\title{
NATURAL AFLATOXICOSIS IN NEONATAL CALVES IN A DAIRY HERD - PATHOLOGICAL DIAGNOSIS
}

\author{
H. HAMALI ${ }^{1}$, J. ASHRAFI-HELAN ${ }^{2} \&$ M. KHORDADMEHR ${ }^{2}$ \\ ${ }^{1}$ Department of Clinical Sciences, Faculty of Veterinary Medicine, University of \\ Tabriz, Tabriz, Iran; ${ }^{2}$ Department of Pathobiology, Faculty of Veterinary Medicine, \\ University of Tabriz, Tabriz, Iran
}

\section{Summary}

Hamali, H., J. Ashrafi-Helan \& M. Khordadmehr, 2021. Natural aflatoxicosis in neonatal calves in a dairy herd - pathological diagnosis. Bulg. J. Vet. Med., 24, No 2, 291-296.

\begin{abstract}
Aflatoxins have been known as an important cause of animal disease for 30 years. The toxins occur naturally on animal feeds either in the field or during storage when moisture content and temperatures are sufficiently high for mould growth. The present paper describes clinical signs besides of gross and histopathological features of aflatoxicosis in 2 to 5-day-old dairy calves which were infected naturally in February 2017. According to the history and clinical examinations, clinical signs included anorexia, ataxia, reluctance to move, convulsion and neonatal mortality in 2 to 5-day-old calves in this herd. Also, black mould as Aspergillus niger was observed in storage animal feeds especially in rouphage and silage. At necropsy, there was sanguineous ascites with multifocal haemorrhages under serosal and mucosal surfaces of gastrointestinal tract, mesenteric lymph nodes, kidneys, and meninges. The liver and spleen were slightly enlarged and hyperaemic. Intramural oedema and haemorrhage were seen in the wall of gall bladder. Histopathological examinations revealed haemorrhagic enteritis, abomasitis and nephritis. There were multifocal large extramedullary haematopoiesis especially with accumulation of megakaryocytes in the liver and spleen. Moreover, the liver showed mild fatty changes in hepatocytes, centriacinar fibrosis with bile ductules proliferation. In the brain, there were perivascular and perineuronal oedema, and perivascular haemorrhage. According to these results, it seems that calves in this herd suffered from hepatoencephalopathy due to simultaneous acute and subacute aflatoxicosis in which aflatoxins originally produced in animal feeds and transferred during pregnancy and also postnatally via milk, were the cause of neonatal mortality in the herd.
\end{abstract}

Key words: aflatoxin, Aspergillus niger, pathomorphology, hepatic fibrosis, neonatal calves

Aflatoxins are toxic, carcinogenic, mutagenic, teratogenic and immunosuppressive byproducts of Aspergillus spp. that contaminate a wide range of crops and also render the produce unfit for consumption and harmful to human and livestock health (Bhatnagar-Mathur et al., 2015). Toxicity levels that produce clini- 
cal signs vary with the species. Sheep, adult cattle, and rats are quite resistant to the toxin, whereas dogs, pigs, calves, mice, and ducklings are sensitive and may be fatally intoxicated by a dose rate of less than $1 \mathrm{mg} / \mathrm{kg}$ body weight (Stalker \& Hayes, 2007). Briefly, beef and dairy cattle are more susceptible to aflatoxicosis than sheep or horses. Young animals of all species are more susceptible than mature animals to the effects of aflatoxin. Pregnant and growing animals are less susceptible than young animals, but more susceptible than mature animals. Nursing animals may be affected by exposure to aflatoxin metabolites secreted in the milk (Dhanasekaran et al., 2011).

Aflatoxins are metabolised by the hepatic mixed-function oxidase system (MFO) to various toxic and nontoxic metabolites, the proportions of which vary with the species and age of animal involved (Stalker \& Hayes, 2007). The interference with cellular protein synthesis causes many lesions throughout the animal, with the most evident changes being in the liver. Indeed, the main target organ is the liver and the animals suffering from aflatoxicosis show signs of inappetence, depression, fever, tremor, ataxia and cough. Necropsy findings include a yellow-brown liver with centrilobular necrosis, icterus, haemorrhage (Caloni \& Cortinovis, 2011). Histopathological examination of the liver indicated that hepatocytes were notably enlarged, particularly in the centrilobular areas and occasional hepatocyte nuclei were up to five times the diameter of their companions. Hepatocyte cytoplasm was finely vacuolated, many of these vacuoles containing fat (Dhanasekaran et al., 2011).

Four aflatoxins were isolated initially and were identified as B1, B2, GI, and G2 based on their blue or green fluorescence properties and migration patterns during chromatography. The potency and carcinogenicity of these aflatoxins are dependent on the species, dose of intake, duration of intake, age of animal, and nutritional state, but it is generally agreed that $\mathrm{B} 1$ is the most potent of the aflatoxins (Pier, 1981). The economic consequences of aflatoxicosis on young growing animals are substantial and varied.

Aflatoxins cause clinical illness and death when consumed in high quantity; at lesser levels they reduce the growth rate and feed efficiency of young animals and they reduce the ability of animals to cope with infections (Pier, 1991). For these reasons, the present paper describes clinical signs besides of gross and histopathological features of aflatoxicosis in 2 to 5-day-old dairy calves exposed to a high dose of aflatoxin placentally during the pregnancy and also, via the milk after the birth.

\section{Case history and clinical signs}

In February 2017, two calves at 2 and 5 days of age from a dairy farm (housed in tie-stalls during the winter season) were presented to the University of Tabriz (Iran) Veterinary Hospital with severe neurological signs such as shouting and restlessness. According to the owner statement, five neonatal calves died during two months and other clinical signs included head pressure, progressive depression and weakness, ataxia, hyperesthesia, anorexia, reluctance to move, convulsion and neonatal mortality in 2 to 5-day-old calves in this herd, although supportive and antibiotic treatment (by $\mathrm{AD}_{3} \mathrm{E}$ vitamin, dexamethasone and enrofloxacin) was provided. Necropsy was performed, gross morphology was evaluated and the appropriate samples were collected from different tissues (heart, spleen, lung, liver, 

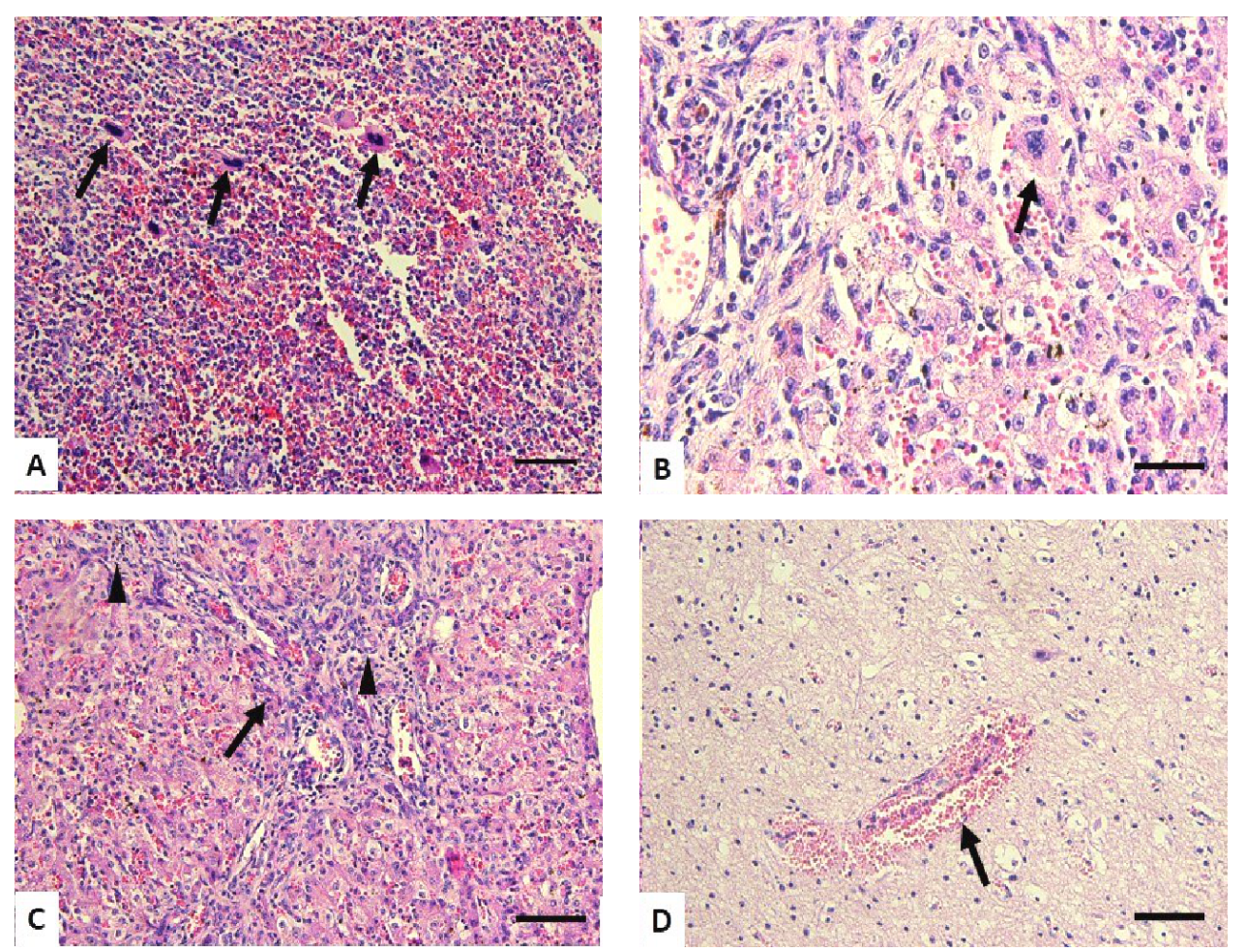

Fig. 1. Aflatoxicosis, 2 day-old calf. A: accumulation of megakaryocytes (arrows) in the spleen (scale bar $=200 \mu \mathrm{m}$ ); B. Accumulation of megakaryocytes (arrow) in the liver (scale bar $=60 \mu \mathrm{m}$ ); . The liver showed mild fatty changes in hepatocytes with bile duct proliferation (arrow heads) hepatic fibrosis (arrow) (scale bar=200 $\mu \mathrm{m}$ ); D. In the brain, there were perivascular and perineuronal aedema, and haemorrhage (arrow) (scale bar $=200 \mu \mathrm{m}$ ); H \& E.

intestine, kidney and brain) for histopathological studies. The tissue samples were fixed in $10 \%$ neutral buffered formalin, processed routinely, and embedded in paraffin wax. Sections $(5 \mu \mathrm{m})$ were stained by haematoxylin and eosin and studied by a light microscope (CH-30, Olympus, Japan).

At necropsy, there was sanguineous ascites with multifocal haemorrhages under serosal and mucosal surfaces of gastrointestinal track, mesenteric lymph nodes, kidneys, and meninges. The liver and spleen were slightly enlarged and hyperemic. Intramural oedema and hae- morrhage were seen in the wall of gall bladder. Histopathological examinations of gastrointestinal tract revealed severe haemorrhagic enteritis and abomasitis with oedema and hyperaemia. Multifocal nephritis associated with renal tubular epithelial degeneration and detachment with cast formation were observed in kidney. There was multifocal large extramedullary haematopoiesis especially with accumulation of megakaryocytes in the spleen and liver (Fig. 1A, B). Moreover, the liver shown mild fatty changes in hepatocytes with bile duct proliferation (Fig. 1C), single cell necrosis and atypical 
hepatocytes. Reticulin and collagen deposition occurred throughout the acinus according to no distinct pattern. In the brain, there were perivascular and perineuronal oedema, and haemorrhage (Fig. 1D). Regarding the case history, clinical observations and necropsy and importantly histopathological findings, aflatoxicosis was reported as a definitive diagnosis.

Mycotoxicosis denotes toxic conditions in livestock and possibly humans because of ingestion of mycotoxincontaminated food which mainly occurs sporadically. Aflatoxins could be produced at appreciable levels in specific conditions of moisture and feed substrate. Moulds capable of synthesising mycotoxins have been recognised on corn, oats, wheat, barley, cottonseed, and peanuts especially in stored conditions (Pier, 1991; Bhatnagar-Mathur et al., 2015). The effect of mycotoxins on animal food has only recently been considered. Economic losses have only been speculated, but may be significant. The clinical signs are almost non-specific, and chronicity is the rule (Nibbelink, 1986; Dhanasekaran et al., 2011).

Since the necropsy findings and histopathology are often helpful for possible diagnosis of aflatoxicosis on basis of clinical signs and history, those were performed carefully. In the present study, as regards the severe nervous clinical signs, we firstly suspected BVD (bovine viral diarrhoea) infection. At necropsy however, there were no gross lesions of BVD such as cerebral hypoplasia in calves. A previous study reported that acute toxicosis with massive doses would lead to death due to hepatic failure with no overt clinical signs. However, acute signs including anorexia, depression, ataxia, and epistaxis or melena (Buck et al., 1976) were similarly observed in the presented cases. Also, in acute clinical aflatoxicosis, signs of acute hepatic injury are reported as coagulopathy, increased capillary fragility, haemorrhage, and prolonged clotting times. Death of the animal may occur within hours or a few days (Pier, 1991; Bhatnagar-Mathur et al., 2015) as observed in the present neonatal calves.

For more information, additional research was done in this herd and black mould as Aspergillus niger was observed in stored animal feeds especially in rouphage and silage because of high moisture of tie-stall in the winter. In histopathological examination, haemorrhagic enteritis, abomasitis and nephritis were observed suggesting a systemic haematogenous disorder. Moreover, there was multifocal large extramedullary haematopoiesis especially with accumulation of megakaryocytes in the liver and spleen which can suggest an anaemic condition. Also, the liver showed mild fatty changes in hepatocytes, centriacinar fibrosis with bile ductules proliferation which can occur in hepatic toxicity. As earlier described, aflatoxins are metabolised by the hepatic MFO system (Stalker \& Hayes, 2007) and it seems that this was responsible for increased hepatocyte fatty change. Besides, the authors believe that exposure to this toxin can stimulate bile duct proliferation in the affected liver after postpartum period. In the brain, there were perivascular and perineuronal oedema, and perivascular haemorrhage. Similar histological lesions were recorded previously (Nibbelink, 1986). At higher doses of aflatoxin, most periacinar hepatocytes disappeared and were replaced by mixture of inflammatory cells, fibroblasts, and primitive vascular channels (Stalker \& Hayes, 2007). In chronic aflatoxin poisoning, most of the effects are still referable to hepatic injury, but on a milder scale. 
The most sensitive clinical sign of chronic aflatoxicosis is reduced rate of growth of young animals (Pier, 1991; BhatnagarMathur et al., 2015). The other lesions of aflatoxicosis are nephrotoxic lesions, immunosuppression, and carcinogenicity (Nibblelink, 1986; Dhanasekaran et al., 2011).

Effects of aflatoxin consumption are similar in all animals; the susceptibility of animals to aflatoxin, however, varies by species, age, and individual (Pier, 1987). Aflatoxin appears to have no direct effect on the foetus in a gestating animal, but foetal changes may be secondarily observed due to maternal toxic biochemical changes and hypovitaminosis A (Bodine $\&$ Mertens, 1983). It was reported that milk fed to ducklings from aflatoxin-fed cattle produced liver lesions identical to those produced by aflatoxin B1 (Allcroft \& Carnaghan, 1963). Another previous study stated that aflatoxin can suppress the immune system of young animals by in utero transfer across the placenta of the pregnant dam (Pier, 1991; BhatnagarMathur et al., 2015). Therefore, it seems that aflatoxin produced by feeding pregnant cattle with stored food contaminated with Aspergillus niger could be transfered across the placenta of the pregnant dam during the pregnancy to the foetus. In addition, neonatal calves were fed milk containing aflatoxin B1. As a result, both mechanisms can result in hepatoencephalopathy with hepatic failure and nervous clinical sings in affected neonatal calves. Therefore, it is suggested to control mould growth in storage facilities through refrigeration, drying, or application of mould inhibitors. Even though complete inhibition of mould growth is very difficult and expensive especially in rainy and growing seasons.
In conclusion, such kind of natural cases in neonates can be encountered incidentally in herds. Bile duct proliferation among findings is highly outstanding. This show us that the cells might have been exposed to aflatoxin formerly i.e. during the in utero period because the progression time was unsufficient for development. We presume that placental transfer of toxin to the foetus might have initiated this proliferation after acute-subacute exposure. So, we consider that newly described findings in detail will be helpful to clinicians and researchers in terms of neonatal intoxications.

\section{ACKNOWLEDGEMENTS}

The authors are grateful to the Faculty of Veterinary Medicine, University Tabriz, Tabriz, Iran for the financial support.

\section{REFERENCES}

Allcroft, R. \& R. B. A. Carnaghan, 1963. Groundnut toxicity: An examination for toxin in human food products from animals fed toxic groundnut meal. The Veterinary Record, 75, 259-263.

Bhatnagar-Mathur, P., S. Sunkara, M. Bhatnagar-Panwar, F. Waliyar, K. K. Sharma, 2015. Biotechnological advances for combating Aspergillus flavus and aflatoxin contamination in crops. Plant Sciences, 234, 119-132. doi: 10.1016/j.plantsci. 2015.02.009.

Bodine, A. B. \& D. R. Mertens, 1983. Biological effects of aflatoxin. In: Aflatoxin and A. flavus in Corn. Auburn University, Alabama Agricultural Experiment Station Research Bulletin, 279, 46-50.

Buck, W. B., G. D. Osweiler \& F. A. Van Gelder, 1976. Clinical and Diagnostic Veterinary Toxicology, $2^{\text {nd }}$ edn, Dubuque, $1 \mathrm{~A}$, Kendall-Hunt Publishing Co. 
Natural aflatoxicosis in neonatal calves in a dairy herd - pathological diagnosis

Caloni, F. \& C. Cartinovis, 2011. Toxicological effects of aflatoxins in horses. The Veterinary Journal, 188, 270-273.

Dhanasekaran, D., S. Shanmugapriya, N. Thajuddin \& A. Panneerselvam, 2011. Aflatoxins and Aflatoxicosis in Human and Animals, In: Aflatoxins - Biochemistry and Molecular Biology, ed Ramón Gerardo Guevara-González, IntechOpen.

Nibbelink, S. K., 1986. Aflatoxicosis in food animals: A clinical review, Iowa State University Veterinarian, 48, 28-31.

Pier, A. C., 1981. Mycotoxins and animal health. In: Advances in Veterinary Science and Comparative Medicine. Academic Press, New York. pp. 185-243.

Pier, A. C., 1987. Aflatoxicosis and immunosuppression in mammalian animals. In: Aflatoxin in Maize, eds M. S. Zuber, E. B. Lillehoj \& B. L. Renfro, CIMMYT, Mexico, pp. 58-65.

Pier, A. C., 1991. The influence of mycotoxins on the immune system. In: Mycotoxins and Animal Food,. eds J. E. Smith \& R. S.
Henderson, CRC Press, Boca Raton, FL, pp. 489-497.

Stalker, M. G. \& M. A. Hayes, 2007. Liver and biliary system (chronic hepatotoxicity), In: Pathology of Domestic Animals, $5^{\text {th }}$ edn, ed M. G. Maxie, pp. 370-371.

Paper received 26.05.2019; accepted for publication 03.08.2019

\section{Correspondence:}

Dr. Monireh Khordadmehr Department of Pathobiology Faculty of Veterinary Medicine University of Tabriz, Tabriz, Iran P.O. Box: 71345-1731 tel: $+98-4136378743$ fax: +98-4136378744

email: khordadmehr@tabrizu.ac.ir 